Génét. Sél. Evol., 1985, 17 (4), 459-480

\title{
Minimisation des coefficients de consanguinité moyens dans les petites populations d'animaux domestiques
}

\author{
H. DE ROCHAMBEAU et C. CHEVALET * \\ I.N.R.A., Station d'Amélioration génétique des Animaux, \\ * Laboratoire de Génétique cellulaire, Centre de Recherche de Toulouse \\ B.P. 27, F 31326 Castanet Tolosan Cedex
}

\begin{abstract}
Résumé
On décrit une méthodologie pour contrôler la consanguinité dans des petites populations d'animaux domestiques, en tenant compte des contraintes d'élevage. On considère une population divisée en groupes de reproduction comprenant un ou plusieurs mâles et des femelles. Un groupe est divisé en classes d'âge, chaque sous-ensemble de la population, défini par le sexe, le groupe de reproduction, et la classe d'âge est décrit par 3 nombres qui désignent l'effectif, les réformes et le renouvellement. La structure génétique est décrite par les coefficients de consanguinité et de parenté moyens calculés sur ces sous-ensembles de la population.

Des conditions sont établies pour minimiser l'accroissement du coefficient de consanguinité pendant les premières générations : (1) le nombre de groupes est supérieur à 10 ; (2) le schéma d'accouplement comporte la circulation des mâles de chaque groupe sur les femelles de tous les autres groupes; (3) le nombre de mâles effectivement utilisés chaque année est aussi grand que possible et leur rythme de renouvellement est rapide. La démarche est illustrée dans le cas de 3 populations composées respectivement de 80 mâles et 2000 femelles, 44 mâles et 616 femelles, 20 mâles et 80 femelles.

Ces règles provoquent une réduction de la variabilité des coefficients de consanguinité moyens. Une structure génétique induite par le schéma d'accouplement se substitue à la structure initiale. Pour des populations de quelques dizaines de mâles et de quelques centaines de femelles, le respect de ces règles permet de limiter l'augmentation du coefficient de consanguinité à 0,3 p. 100 par génération.
\end{abstract}

Mots clés : Coefficients de consanguinité, schéma d'accouplements, animaux domestiques, conservation des ressources animales.

\section{Summary}

Minimizing inbreeding rates in small populations of domestic species

A method is proposed for controlling inbreeding rates in small populations, taking account of usual breeding constraints. A population is divided into breeding groups; each group is divided into age classes, the demographic structure of which are defined by parameters stating their rates of renewal and their reproducing capabilities. At any time, the genetic description is made up of the set of mean inbreeding coefficients of the subsets (sex $\times$ breeding group $\times$ age) and of mean kinship coefficients between these subsets. 
General conditions are given, that minimize inbreeding rates over the first few generations : (i) the number of breeding groups should be at least 10 ; (ii) the mating scheme should involve the circulation of males from any group over females of all other groups; (iii) the number of males used per year, and their rate of turnover should be maximised.

The approach is illustrated by considering three model populations made up of 80 and 2000,44 and 616, 20 and 80 males and females respectively. The proposed rules lead to a reduction in the variance of inbreeding coefficients, and induce a genetic structure independent of the initial relationships between founder individuals. With such rules, the rate of inbreeding does not exceed 0.3 p. 100 per generation, for populations with a few tens of males and a few hundreds of females.

Key words : Inbreeding coefficients, mating scheme, smal populations, domestic animals, conservation of animal resources.

\section{Introduction}

La mise en place de programmes de conservation dans diverses races d'animaux domestiques (DevillaRd, 1980) pose le problème du maintien de la variabilité génétique dans les populations d'effectif limité (YAMADA, 1980). Les populations d'animaux domestiques sont soumises à des contraintes spécifiques. Ces contraintes sont d'abord d'ordre démographique : tous les individus n'ont pas la même probabilité d'avoir un descendant d'un sexe donné, les générations sont chevauchantes, la structure démographique des diverses cohortes est variable dans le temps et entre les élevages qui détiennent les animaux. Par ailleurs, le fait que les animaux en conservation soient dans des élevages et non dans des stations expérimentales limite les choix techniques possibles : la recherche d'une certaine rentabilité interdit par exemple de trop diminuer le nombre de femelles par mâle. Enfin les contraintes sont d'ordre génétique : les animaux de départ sont consanguins et apparentés, l'existence d'élevages empêche la réalisation d'accouplements aléatoires au niveau de l'ensemble de la population, sauf en cas de recours exclusif à l'insémination artificielle.

Dans ces conditions, les résultats théoriques concernant la maîtrise de la dérive génétique dans les populations d'effectifs limités ne sont pas directement applicables. Le calcul d'une taille efficace tient compte du chevauchement des générations (KIMURA \& CRow, 1963 a ; Hill, 1972), mais ne permet pas de comparer divers systèmes d'accouplements entre plusieurs groupes de reproduction. Par ailleurs, les schémas d'accouplements circulaires entre quelques individus (WRIGHT, 1921 ; KIMURA \& CROW, 1963 b ; CocKerham, 1970) supposent la séparation des générations et la constance des effectifs. Pour minimiser la dérive génétique dans une population animale, en respectant les contraintes d'élevage, nous proposons d'une part un cadre général assez souple pour décrire la structure d'une population et ses variations dans le temps, et d'autre part une démarche semi-empirique pour rechercher des systèmes qui optimisent l'évolution probable de la structure de cette population.

Dans une première partie nous définissons un ensemble de paramètres pour caractériser la démographie et le schéma d'accouplement, et nous indiquons comment ces paramètres permettent de calculer des critères mesurant la dérive génétique, et de simuler l'évolution d'une population. Nous illustrons ensuite notre démarche avec 3 exemples de populations, où nous analysons les effets du mode de circulation des mâles entre élevages, de la structure démographique et des variations de la structure génétique initiale, sur l'évolution du taux de consanguinité. Nous montrons aussi comment des résultats théoriques peuvent guider la recherche de systèmes 
optimaux, mais aussi que leur transposition directe aux populations d'animaux domestiques est en général incorrecte et peut même induire en erreur.

\section{Méthodes de description des populations}

\section{A. Structure démographique et schéma d'accouplement}

Nous considérons une population présentant une structuration en groupes de reproduction. Ces groupes peuvent représenter des élevages, ou des familles d'animaux réunis selon leur apparentement : le problème de la constitution de ces groupes selon les informations généalogiques (Rochambeau, 1983) ne sera pas abordé ici, nous évoquerons seulement l'incidence des parentés initiales entre animaux et entre groupes sur l'efficacité d'un schéma d'accouplement. Cette structuration en groupes traduit la répartition spatiale des animaux, en négligeant les migrations entre élevages : pratiquement on considérera qu'un jeune est élevé dans l'élevage où il est né, de sorte que ces groupes de reproduction se perpétuent par ascendance maternelle (les groupes de reproduction peuvent être considérés comme des «matronymes »). Enfin, la population est considérée en des instants successifs, séparés par un intervalle correspondant au rythme de reproduction de l'espèce. Pour des ovins ou des caprins par exemple, l'intervalle est d'une année, et nous supposons que les recensements sont effectués à une date proche du début des mises bas; les individus les plus jeunes, dans leur première année (non révolue), constituent la classe d'âge numéro 1.

A un instant donné (t), la population est alors décrite par une suite de nombres $\mathbf{n}_{\mathrm{ijk}}$ désignant les effectifs d'animaux dans chaque «cellule », définie par le sexe $(i)(i=1,2)$, le groupe de reproduction $(j)(j=1,2, \ldots, G)$, et la classe d'âge (k) $\left(\mathrm{k}=1,2, \ldots, \mathrm{C}_{\mathrm{i}}\right)$. Pour être dynamique, cette description est complétée de façon à ce que l'on puisse en déduire la constitution de la population à l'instant suivant $(t+1)$. En se limitant au cas où les animaux demeurent d'un instant à l'autre dans le même élevage, et donc dans le même groupe, on doit indiquer parmi les $\mathbf{n}_{\mathrm{ijk}}(\mathrm{t})$ individus de la cellule (ijk) à l'instant $(t)$, l'effectif $p_{i j k}(t)$ de ceux qui ne seront pas réformés, de telle sorte qu'ils se retrouveront à l'instant suivant dans la cellule $(\mathrm{i}, \mathrm{j}, \mathrm{k}+1)$ puisqu'ils auront vieilli d'une unité de temps :

$$
\begin{aligned}
& \mathrm{n}_{\mathrm{ijk}}(\mathrm{t})=\mathrm{p}_{\mathrm{ijk}}(\mathrm{t})+\text { (réformes) } \\
& \mathrm{n}_{\mathrm{ij}(\mathrm{k}+1)}(\mathrm{t}+1)=\mathrm{p}_{\mathrm{ijk}}(\mathrm{t})
\end{aligned}
$$

Si l'on réunit dans la dernière classe d'âge $\left(k=C_{i}\right)$ les animaux d'âges égal ou supérieur à $C_{i}$, un paramètre $\mathrm{p}^{\prime}{ }_{\mathrm{ijc}}$ doit être défini, désignant le nombre d'animaux de cette cellule $\left(i, j, C_{i}\right)$ qui ne sont pas réformés et qui se trouveront à l'instant suivant dans la cellule homologue :

$$
\begin{aligned}
& \mathrm{n}_{\mathrm{ijc}}(\mathrm{t})=\mathrm{p}_{\mathrm{ijc}}^{\prime}(\mathrm{t})+\text { (réformes) } \\
& \mathrm{n}_{\mathrm{ijc} \mathrm{C}_{\mathrm{i}}}(\mathrm{t}+1)=\mathrm{p}_{\mathrm{ij \textrm {C } _ { i }}}^{\prime}(\mathrm{t})+\mathrm{p}_{\mathrm{ij}\left(\mathrm{C}_{\mathrm{i}}-1\right)}(\mathrm{t})
\end{aligned}
$$

Cette simplification correspond, pour cette ultime classe d'âges, à un âge moyen égal à : $\mathrm{C}+\mathrm{p}^{\prime} / \mathrm{p}$. 
Ces relations décrivent le vieillissement de la population, d'autres doivent exprimer l'introduction des jeunes, c'est-à-dire la constitution des cellules ( $i, j, 1)$ désignant à chaque instant la cohorte des jeunes de remplacement. D'une façon générale cette constitution peut être précisée par des relations du type :

$$
\mathrm{n}_{\mathrm{ij} 1}(\mathrm{t}+1)=\mathrm{R}_{1 \mathrm{j}_{1} \mathrm{k}_{1} ; 2 \mathrm{j}_{1}^{\prime} \mathrm{k}_{1}^{\prime}{ }_{1}}(\mathrm{t})+\ldots+\mathrm{R}_{1 \mathrm{j}_{\mathrm{q}} \mathrm{k}_{q} ; 2 \mathrm{j}_{q^{\prime}} \mathrm{k}^{\prime}{ }_{q}}(\mathrm{t})
$$

signifiant que parmi les $n_{i j 1}(t+1)$ jeunes de la cellule (ij1), un nombre $R_{1 j_{1} k_{1} ; 2 j^{\prime} k^{\prime}}(t)$ provient d'accouplements entre individus des cellules $\left(1, \mathrm{j}_{1}, \mathrm{k}_{1}\right)$ et $\left(2, \mathrm{j}_{1}^{\prime}, \mathrm{k}^{\prime}{ }_{1}\right)$ de l'ins-

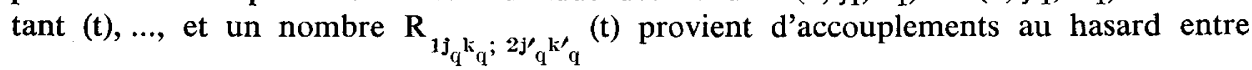
individus des cellules $\left(1, \mathrm{j}_{\mathrm{q}}, \mathrm{k}_{\mathrm{q}}\right)$ et $\left(2, \mathrm{j}^{\prime}{ }_{\mathrm{q}}, \mathrm{k}_{\mathrm{q}}^{\prime}\right)$ de l'instant $(\mathrm{t})$. On admet alors que la probabilité qu'un individu participe à la reproduction dépend seulement de son âge et de son sexe. Ce mode de reproduction constitue une sorte de panmixie restreinte, analogue à celui rencontré dans les modèles de migration (MALEcot, 1972).

Pratiquement, on convient que les descendants d'une femelle d'un groupe (j) sont élevés dans ce même groupe (j) (on a donc ici $\mathrm{j}^{\prime}{ }_{1}=\ldots \mathrm{j}_{\mathbf{q}}=\mathrm{j}$ ), et que les femelles d'un groupe (j) sont saillies uniquement par les mâles d'un seul groupe $\left(j^{*}\right)$, fonction de $(j)$ et de $(t)$. La spécification de $n_{i j 1}(t+1)$ peut alors s'écrire :

$$
\mathrm{n}_{\mathrm{ij} 1}(\mathrm{t}+1)=\mathrm{R}_{1 \mathrm{j}^{* k_{1}} ; 2 \mathrm{jk}_{1}^{\prime}}(\mathrm{t})+\ldots+\mathrm{R}_{1 \mathrm{j}^{* k_{\mathrm{q}}} ; 2 \mathrm{jk}_{\mathrm{q}}^{\prime}}(\mathrm{t})
$$

Le nombre :

$$
b_{i, 2, j, k^{\prime}}(t)=\sum_{k} R_{1 j^{*} k ; 2 j k^{\prime}}(t)
$$

est le nombre de descendants du sexe $(i), i=1,2$, issus des femelles de la cellule $\left(2, j, k^{\prime}\right)$ à l'instant $(t)$. De même le nombre de descendants du sexe (i), $i=1,2$, issus des mâles de la cellule $\left(1, \mathrm{j}^{*}, \mathrm{k}\right)$ à l'instant $(\mathrm{t})$ est :

$$
b_{i, 1, j^{* k}}(t)=\sum_{k^{\prime}} R_{1 j^{*} k ; 2 j k^{\prime}}(t)
$$

Si de plus dans un couple de reproducteurs l'âge du partenaire de sexe (i') fixe l'âge du partenaire de sexe opposé, la donnée des nombres $b_{i, i^{\prime}, j, k}(t)$ est alors suffisante pour fixer les $n_{i j 1}(t)$.

A chaque cycle de la reproduction, un schéma de circulation des mâles est décrit par une relation de la forme :

$$
\mathrm{j}^{*}=\mathrm{j}-\mathrm{d}(\mathrm{t}) \quad(\text { modulo } \mathrm{G})
$$

où $\mathrm{d}(\mathrm{t})$ est une fonction périodique du temps à valeurs entières. Lorsque cette fonction est constante, nous parlons de schéma "sans décalage », que nous opposons aux schémas avec décalage pour lesquels la fonction $d$ a une période égale à $(\mathrm{G}-1)$. Un tel schéma se définit par les $(\mathrm{G}-1)$ premières valeurs prises par $d$. Ces valeurs sont alors choisies de façon à retarder le plus possible l'apparition d'accouplement entre des groupes apparentés; elles s'inspirent du schéma défini par WRIGHT sous le nom de «Maximum avoidance of inbreeding (WRIGHT, 1921) et perfectionné par d'autres auteurs (Kimura \& Crow, $1963 \mathrm{~b}$; CocKerham, 1970). 
Ce type de description permet d'étudier des situations complexes. On peut faire varier les paramètres démographiques des différents groupes de reproduction (RochamBEAU et al., 1979). On peut aussi utiliser cette description pour étudier des problèmes démographiques ou génétiques (Vu TIEN KHANG, 1983) comme la diffusion des gènes transmis par des individus sélectionnés dans les différentes strates d'une population. Par ailleurs la description comportant de façon explicite les effectifs d'animaux dans chaque groupe, elle peut être utilisée directement pour étudier les fluctuations aléatoires dues à l'échantillonnage, et peut constituer un cadre pour prévoir dans des cas concrets, les conséquences de ces fluctuations d'échantillonnage sur la variabilité de la réponse à la sélection.

\section{B. Caractérisation de la variabilité génétique et de ses modifications et choix d'une démarche}

La variabilité génétique d'une population peut se définir par l'ensemble des fréquences alléliques en de nombreux locus polymorphes. Cependant cette notion demeure une abstraction dans la plupart des espèces domestiques. Néanmoins, si l'on connaissait ces fréquences alléliques en un instant donné, dans les différents groupes d'une population, la connaissance des règles démographiques et de reproduction, décrites par les paramètres définis ci-dessus, permettrait de prévoir en probabilité la structure génétique de la population à un instant ultérieur. Quand on ne dispose pas de telles observations, on doit se limiter à des mesures de l'évolution de cette variabilité génétique. En se restreignant à des gènes neutres, on peut envisager divers critères de cette évolution entre 2 instants.

Pour chacun des groupes constituant la population, on peut calculer les probabilités qu'un gène tiré dans un groupe provienne d'un gène présent à un instant précédent dans le même groupe. Ces probabilités d'origine des gènes permettent de comparer les poids respectifs des différents groupes définis à l'instant initial (JAMES, 1972).

La façon classique de mesurer la perte de variabilité génétique entre 2 instants est de calculer la probabilité que 2 gènes homologues soient copies d'un gène unique présent à l'instant initial. Compte tenu de la description adoptée, on peut définir à chaque instant un coefficient moyen de consanguinité pour chacun des groupes et des coefficients moyens de parentés entre individus, dont le premier appartient à un groupe et le second à un autre groupe. Cet ensemble de coefficients se déduit des quantités analogues définies à l'instant précédent par un système d'équations linéaires dont les coefficients sont fonctions des paramètres démographiques et de reproduction décrivant le passage entre deux instants successifs (Cockerham, 1967 ; CHOY \& WEIR, 1978).

Il existe aussi plusieurs définitions de la taille efficace $\mathrm{Ne}$ d'une population (Kimura \& Crow, 1963 a ; Felsenstein, 1971 ; Kimura \& Ohta, 1971 ; Hill, 1972) qui permettent de caractériser la dérive génétique. Cependant les conditions d'application de ces définitions sont très strictes, et elles ne permettent pas de tenir compte d'un fractionnement de la population en groupes, ni d'échanges de reproducteurs entre groupes, alors que ce type de structure, d'une part s'apparente à celle des petites populations d'animaux réparties entre plusieurs élevages, et d'autre part, constitue une solution théorique au problème du maintien de la variabilité génétique (KIMURA \& Crow, 1963 b). 
Dans cette étude, nous utilisons le calcul des probabilités d'identité entre deux gènes, tirés dans un même groupe d'animaux ou dans 2 groupes d'animaux. Dans la discussion nous préciserons les limites de ce critère et nous évoquerons ce que pourraient apporter d'autres critères.

La démarche poursuivie consiste à rechercher les systèmes de gestion et de reproduction qui minimisent l'accroissement des coefficients de consanguinité, sur un intervalle de temps fixé. A partir de la description de la structure démographique et du schéma d'accouplement, un programme (Rochambeau et al., 1979) génère les équations d'évolution des coefficients de parenté et de consanguinité et les résout. La comparaison de diverses variantes permet de trouver d'une manière semi-empirique les solutions qui concilient une faible augmentation de la consanguinité avec les contraintes auxquelles est soumise la population.

\section{Applications}

Pour illustrer notre démarche, nous considérons 3 populations de tailles et de caractéristiques différentes. Nous étudions les effets du nombre de groupes de reproduction et du mode de circulation des mâles entre les groupes de reproduction, les conséquences des variations de la structure démographique, ainsi que les modifications de la structure génétique.

\section{A. Description de trois populations modèles}

La première comporte 44 mâles et 616 femelles, et correspond à la race caprine Poitevine. La deuxième représente 8 élevages d'une race ovine, Mérinos Précoce, elle comprend 80 mâles et 2000 femelles. La troisième possède 20 mâles et 80 femelles, elle correspond au modèle d'une race de volaille en conservation. Chacune de ces populations est divisée en groupes de reproduction.

Les mâles d'un groupe sont accouplés avec les femelles d'un seul groupe, les mâles et les femelles d'un même groupe ne sont jamais accouplés ensemble; les descendants mâles et femelles d'un groupe restent dans le groupe de leur mère. Dans cette étude nous supposons que la structure démographique est identique dans tous les groupes et demeure stationnaire dans le temps. Les paramètres démographiques des groupes des populations Poitevine et Mérinos Précoce sont donnés aux tableaux 1 et 2 respectivement. Le modèle "Aviaire» se compose de 5 groupes de reproduction, qui comprennent chacun 4 mâles et 16 femelles; ces individus sont renouvelés par moitié à chaque intervalle de temps.

Pour spécifier la circulation des mâles entre les groupes de reproduction nous distinguons les schémas sans décalage et les schémas avec décalage (cf. II.A, formule 9). Dans le cas du modèle Poitevin, nous pouvons avoir par exemple le schéma avec décalage suivant entre 11 groupes :

$$
\begin{aligned}
& \mathrm{d}(1)=1 ; \mathrm{d}(2)=2 ; \mathrm{d}(3)=4 ; \mathrm{d}(4)=8 ; \\
& \mathrm{d}(5)=5 ; \mathrm{d}(6)=10 ; \mathrm{d}(7)=9 ; \mathrm{d}(8)=7 ; \\
& \mathrm{d}(9)=3 ; \mathrm{d}(10)=6 ;
\end{aligned}
$$




\section{TABleau 1}

Paramètres démographiques d'un groupe de reproduction du modèle Poitevin, dans le cas de 11 groupes.

Demographic parameters of a breeding group in the Poitevin model population of goats in the case of 11 groups.

\begin{tabular}{|c|c|c|c|c|c|}
\hline \multicolumn{2}{|c|}{ Paramètres } & \multirow[b]{2}{*}{$\mathbf{n}$} & \multirow[b]{2}{*}{$b_{m}$} & \multirow[b]{2}{*}{$b_{f}$} & \multirow[b]{2}{*}{ p } \\
\hline Sexe & $\begin{array}{c}\text { Age } \\
\text { (année) }\end{array}$ & & & & \\
\hline \multirow{2}{*}{$\begin{array}{c}\text { Mâle } \\
m\end{array}$} & 1 & 2 & 0 & 4 & 2 \\
\hline & 2 & 2 & 2 & 8 & 0 \\
\hline \multirow{4}{*}{$\begin{array}{c}\text { Femelles } \\
f\end{array}$} & 1 & 12 & 0 & 1 & 11 \\
\hline & 2 & 11 & 0 & 3 & 9 \\
\hline & 3 & 9 & 2 & 2 & 8 \\
\hline & 4 & 24 & 0 & 6 & 16 \\
\hline
\end{tabular}

$n$ : taille de sous-groupe; $b_{\mathrm{m}}\left(b_{\mathrm{f}}\right)$ : nombre de descendants mâles (femelles) du sous-groupe; $\mathrm{p}$ : nombre d'animaux survivants l'année suivante; noter que les femelles du sous-groupe 4 sont d'âges supérieurs ou égaux à 4 ans. Les mâles d'un an sont accouplés aux chèvres en première et deuxième lactation; ceux de 2 ans aux chèvres plus âgées, des sous-groupes femelles $n^{\circ} 3$ et 4 . $\mathrm{n}$ : size of the sub-group : $\mathrm{b}_{m}\left(\mathrm{~b}_{f}\right)$ : number of male (female) offspring born out of the sub-group; $p$ : numbers of animals surviving from one year to the next; note that females in sub-group 4 are of age greater than or equal to 4. Males of age 1 are mated to goats of ages 1 and 2 ; males of age 2 are mated to goats of ages 3, 4 and older.

\section{TABLEAU 2}

Paramètres démographiques d'un groupe de reproduction du modèle Mérinos Précoce dans le cas où cette population se compose de 16 groupes (Symbolisme identique au tableau 1).

Demographic parameters of a breeding group

in the Mérinos Précoce model population of sheep, in the case of 16 groups (same symbols as in table 1).

\begin{tabular}{c|c|c|c|c|c}
\hline \hline \multicolumn{2}{c|}{ Paramètres } & & & \\
\hline Sexe & $\begin{array}{c}\text { Age } \\
\text { (année) }\end{array}$ & $\mathrm{n}$ & $\mathrm{b}_{\mathrm{m}}$ & $\mathrm{b}_{\mathrm{f}}$ & $\mathrm{p}$ \\
\hline Mâle & 1 & 10 & 0 & 0 & 4 \\
$m$ & 2 & 4 & 4 & 23 & 4 \\
\hline & 1 & 23 & 0 & 0 & 21 \\
& 2 & 21 & 0 & 4 & 19 \\
Femelles & 3 & 0 & 4 & 17 \\
$f$ & 19 & 3 & 4 & 15 \\
& 17 & 3 & 5 & 13 \\
& 5 & 3 & 6 & 17 \\
\hline
\end{tabular}


puis :

$$
\mathrm{d}[\mathrm{t}+\mathrm{k}(\mathrm{G}-1)]=\mathrm{d}(\mathrm{t}) ; \text { avec } \mathrm{G}=11 .
$$

Cette fonction se note simplement :

$$
d=1,2,4,8,5,10,9,7,3,6 .
$$

Les autres décalages sont spécifiés dans les légendes des figures et tableaux.

Les conditions initiales sont caractérisées par 3 ensembles de valeurs : les coefficients moyens de consanguinité des différentes cohortes, et pour chaque couple de cohortes, les coefficients de parenté moyens entre animaux d'un même groupe de reproduction, et les coefficients moyens de parenté entre animaux de deux groupes de reproduction. Dans les 2 cas des modèles Poitevin et Mérinos Précoce, toutes les cohortes des divers groupes de reproduction ont un même coefficient moyen de consanguinité $F(F=0,02)$; pour ces mêmes modèles nous avons admis que les coefficients moyens de parenté entre 2 cohortes ne dépendaient que de l'appartenance des 2 groupes d'animaux à un même groupe ou à 2 groupes distincts de reproduction, cela définit 2 groupes de valeurs, $T_{o}$ et $T_{d}$ (parentés entre cohortes intra-groupes et entre groupes respectivement, tabl. 3 et 4 ). Ces coefficients moyens de parenté schématisent la façon dont les groupes de reproduction ont été constitués : on a recherché à rassembler dans un même groupe les animaux les plus apparentés. Dans le cas du modèle «Aviaire», les coefficients moyens de consanguinité et de parenté varient de façon quelconque (tabl. 3, 4 et 5).

\section{B. Nombre de groupes de reproduction et circulation des mâles}

Le choix d'un schéma de circulation dépend du nombre et de la taille des groupes de reproduction. Avec le modèle Poitevin, nous avons envisagé 5, 11 ou 23 groupes (fig. 1). Dans le programme de conservation de la race Poitevine, il y avait 11 groupes de reproduction. A partir de là il est possible de réunir ces groupes 2 à 2 pour passer à 5 groupes de reproduction; on peut aussi diviser chaque groupe en 2 pour obtenir 23 groupes de reproduction. La recherche d'un bon schéma avec décalage nous ayant conduit initialement à préférer un nombre impair de groupes de reproduction, ces divisions par 2 n'ont pu être appliquées strictement. Sauf s'il y a peu de groupes de reproduction, qui sont alors de grande taille (ici, 123 femelles pour 5 groupes), un schéma avec décalage s'avère préférable. Parmi les combinaisons étudiées, un tel schéma entre 23 groupes permet d'obtenir le coefficient moyen de consanguinité minimal après 20 ans (2,3 p. 100); sur la même période le pire schéma est le schéma sans décalage entre les mêmes 23 groupes. La supériorité des schémas avec décalage se renforce lorsque la taille des groupes diminue : dans le modèle «Aviaire», un schéma avec décalage entre 5 groupes de 16 femelles est meilleur qu'un schéma sans décalage (fig. 2, comparée à la fig. 1). On observe néanmoins que malgré le faible effectif total de cette population, un accroissement du nombre de groupes est encore, en théorie, souhaitable.

Pour des populations à générations séparées, de mêmes tailles totales, KIMurA \& Crow (1963 b) ont montré la supériorité à long terme du schéma sans décalage avec un fractionnement en de nombreux groupes égaux. Les résultats opposés que 
CONSANGUINITÉ DES POPULATIONS ANIMALES

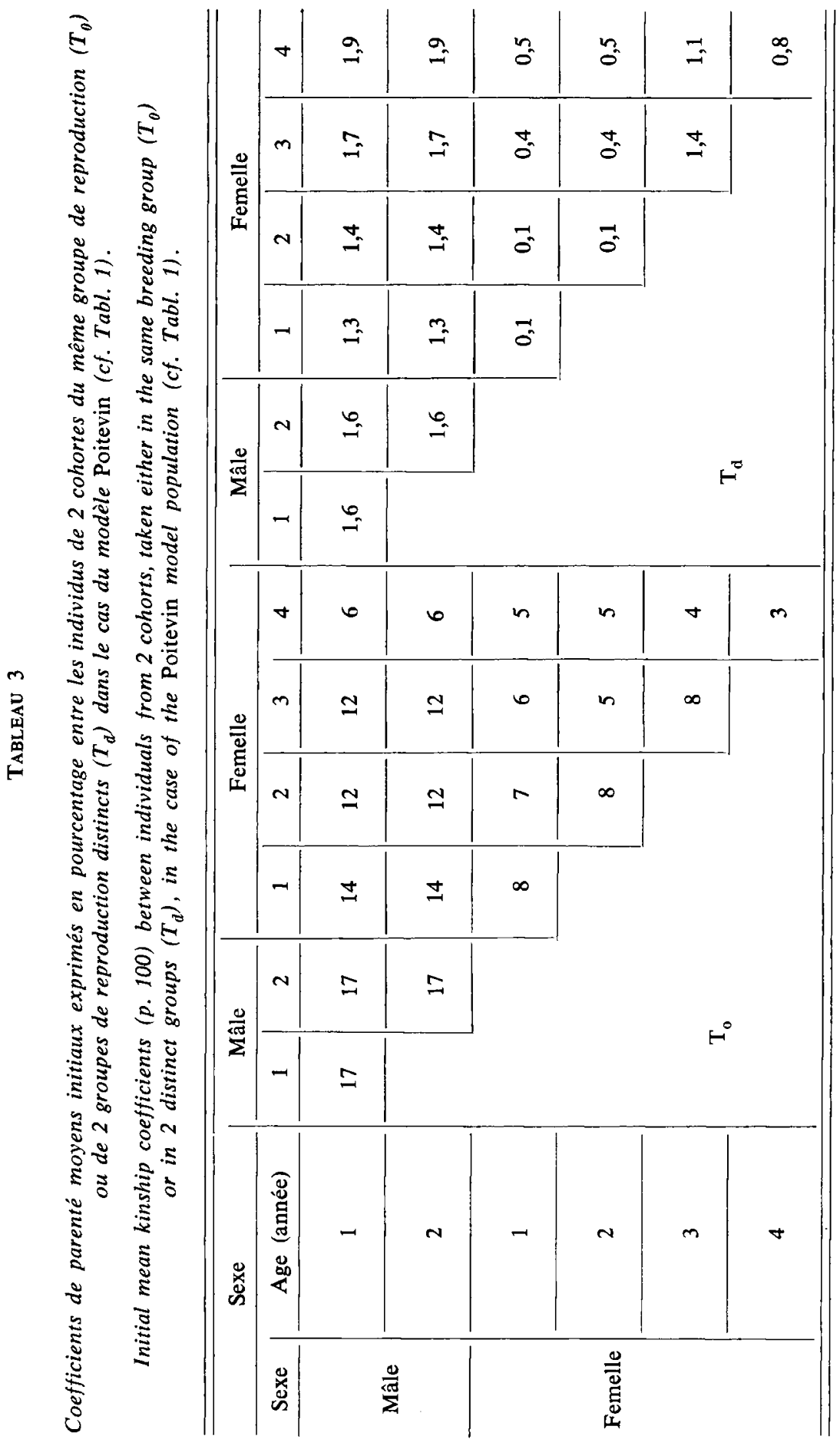




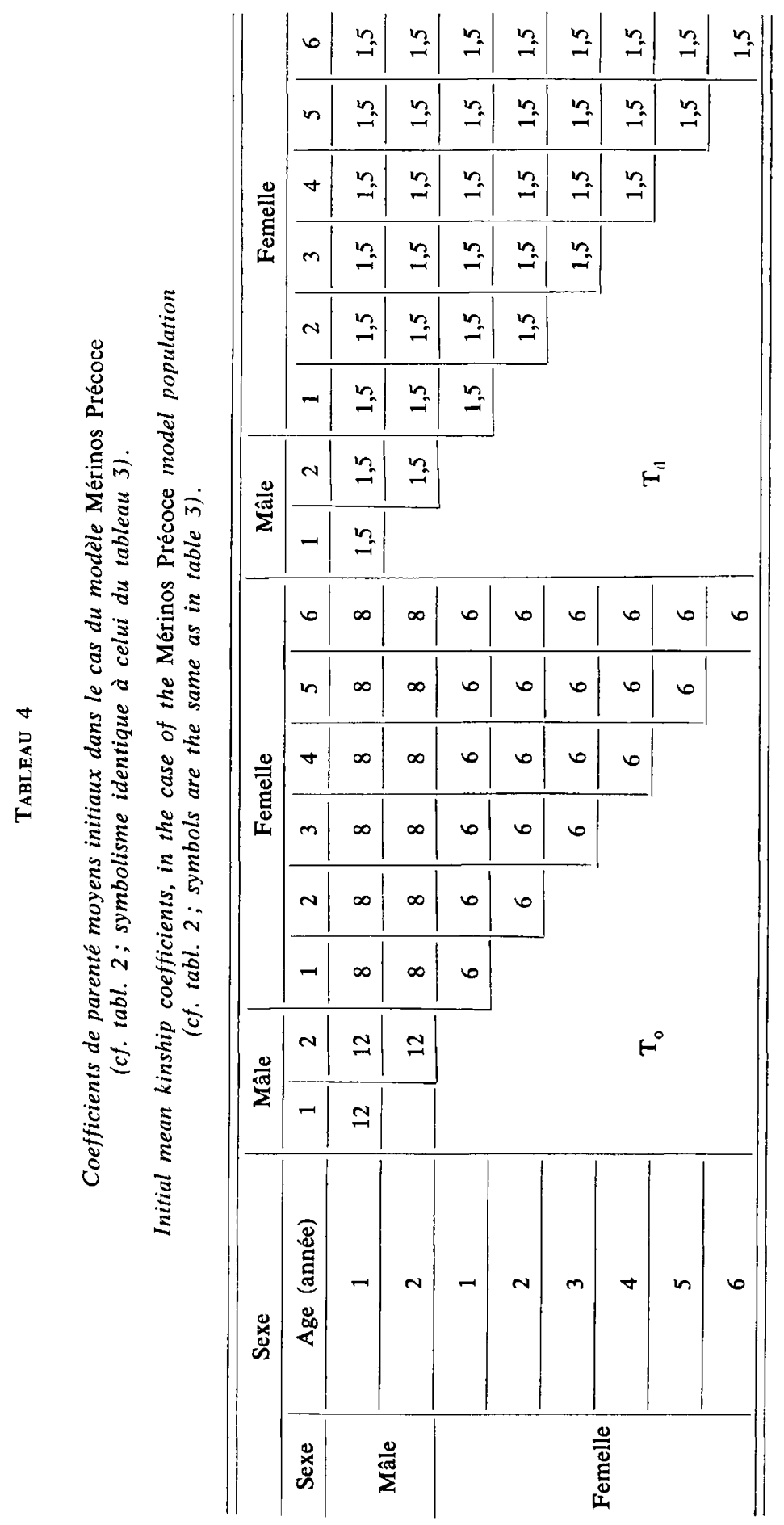




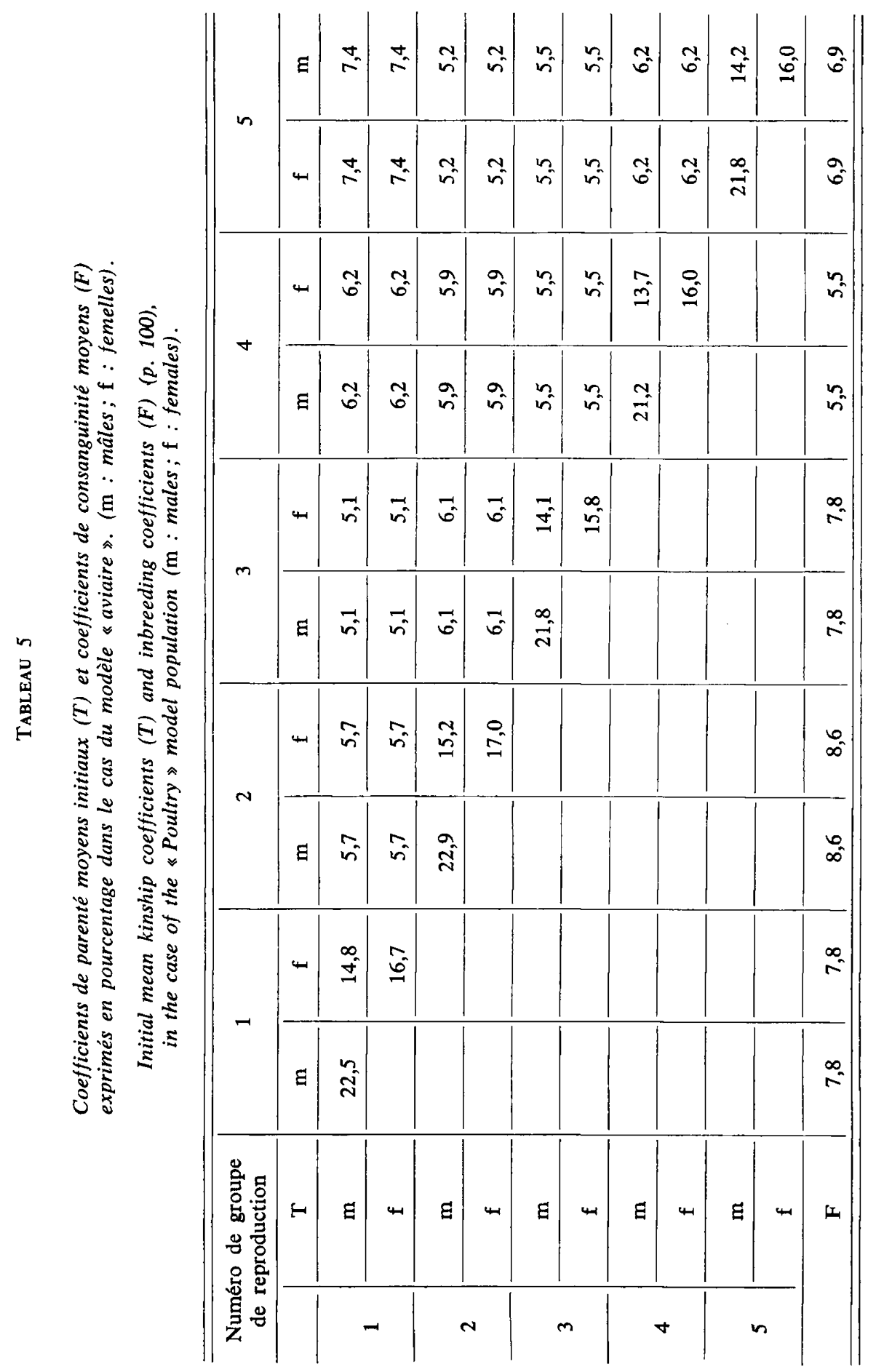




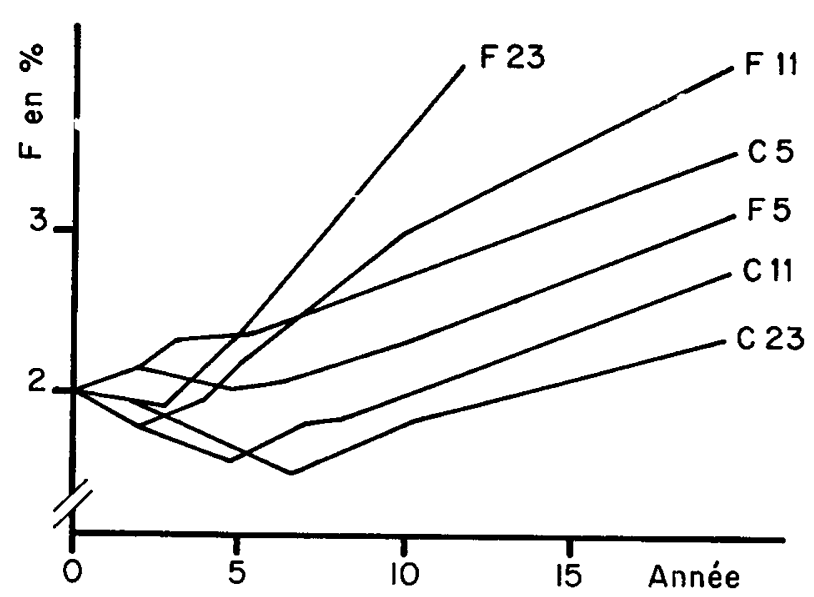

FIG. 1

Evolution de coefficient moyen de consanguinité dans le cas du modèle Poitevin, en fonction du type de schéma d'accouplement

( $F:$ sans décalage, $C:$ avec décalage) et du nombre de groupes $(5,11$ ou 23).

$d=1,2,4,3 ; d=1,2,4,8,5,10,9,7,3,6 ; d=1,2,4,8,16,9,18,13,3,6$, $12,22,21,19,15,7,14,5,10,20,17,11$ respectivement.

Change with time of mean inbreeding coefficient in the case of the Poitevin model population,

as a function of the mating scheme ( $F$ : fixed mating rule; $C$ : circular rule) and of the number of groups $(5,11$ or 23$)$.

$\mathrm{d}=1,2,4,3 ; \mathrm{d}=1,2,4,8,5,10,9,7,3,6 ; \mathrm{d}=1,2,4,8,16,9,18,13,3,6$, $12,22,21,19,15,7,14,5,10,20,17,11$ respectively.

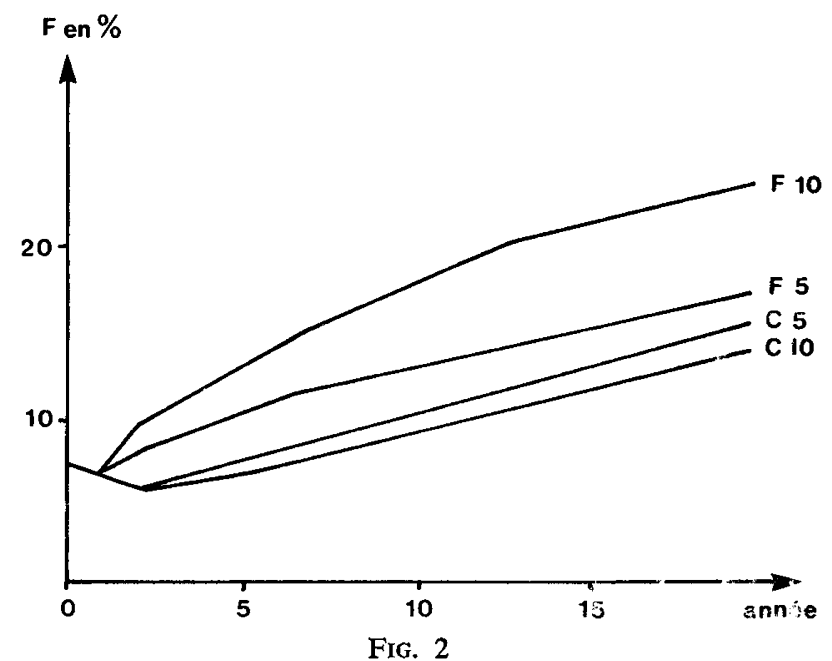

Evolution du coefficient moyen de consanguinité dans le cas du modèle "Aviaire», en fonction du type de schéma de circulation des mâles (F ou $C$ ), et du nombre de groupes de reproduction (5 ou 10).

Change of mean inbreeding coefficient in the case of the "Poultry》 model population, as a function of the mating scheme $(F$ or $C)$ and of the number of groups (5 or 10). 
nous obtenons ne sont pas contradictoires puisqu'ils concernent le court terme : sans décalage l'effet prépondérant est alors l'apparition d'une consanguinité systématique de position, d'autant plus élevée que les groupes sont de petites tailles et donc nombreux. Inversement un schéma avec décalage permet d'éviter les accouplements consanguins d'autant plus longtemps que le nombre de groupes est plus élevé. Mais pour un effectif d'animaux et un nombre de groupes donnés, le meilleur schéma de circulation dépend des caractéristiques démographiques, de l'intervalle de temps considéré, et des conditions initiales; quelques simulations sont alors nécessaires pour obtenir une fonction $d$ satisfaisante.

Il est possible de concevoir des schémas dans lesquels on allonge la période de la fonction de circulation $d$. A partir d'une fonction comme :

$$
\mathrm{d}=1,2, \ldots,
$$

on peut construire un décalage double,

$$
\mathrm{d}^{\prime}=1,1,2,2, \ldots,
$$

où chacun des types d'accouplement prescrits par $d$ est répété 2 fois de suite. L'étude, pour les exemples traités de populations de petits ruminants, de quelques décalages doubles, triples, quadruples et quintuples indique que les décalages simples, doubles et triples s'avèrent comparables sur le plan théorique (fig. 3).

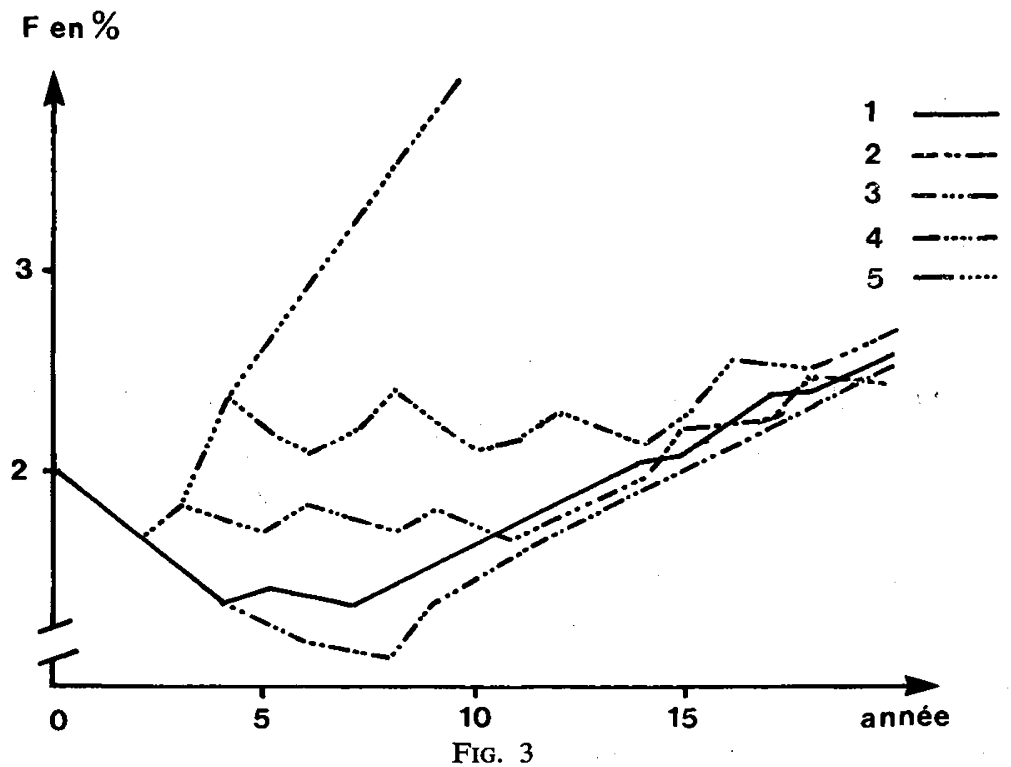

Evolution du coefficient moyen de consanguinité dans le cas du modèle Mérinos précoce, selon le type de schéma.

Change with time of mean inbreeding coefficient

in the case of the Mérinos précoce model population, as a function of the mating rule.

(1) Décalage simple, avec $d=1,2,4,8,15,13,6,12,11,5,3,14,7,10,9$.

Circular matings, with $\mathrm{d}=1,2,4,8,15,13,6,12,11,5,3,14,7,10,9$ (see section $(I-C)$.

(2), (3), (4) Le même décalage avec répétition double, triple et quadruple.

The same rule is applied two, three and four times respectively (see section III-A).

(5) Sans décalage.

Fixed mating rule, $\mathrm{d}=1$. 
L'étude d'autres exemples permet de penser que ces résultats sont généraux pour ce type de population.

\section{Structure démographique}

1. Nombre et renouvellement des reproducteurs mâles

La réduction du nombre de mâles mis en service à chaque cycle induit naturellement un accroissement plus rapide de la consanguinité (fig. 4, cas $n^{\circ} 4$ ).

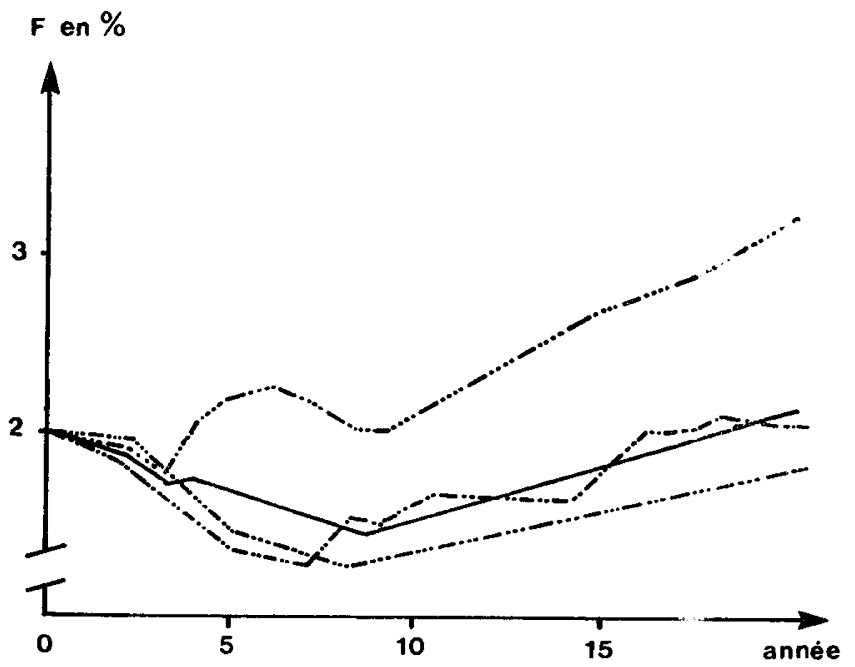

Fig. 4

Evolution du coefficient moyen de consanguinité dans le cas du modèle Poitevin, selon la politique démographique.

Change with time of mean inbreeding coefficient in the case of the Poitevin model population, as a function of the demographic policy.

(1) Référence, cf. tableau 1.

Reference case, see table 1.

(2) Renouvellement rapide des mâles.

Faster turnover of males.

(3) Vieillissement du cheptel femelle.

Older females.

(4) Réduction du nombre de mâles.

Smaller number of males.

Les paramètres modifiés dans les trois derniers cas, par rapport au tableau 1, sont donnés ci-dessous.

Parameters modified in the last three cases are listed below.

Cas - Case

Paramètres modifiés - Modified parameters

(2)

$$
\begin{aligned}
& n(\mathrm{~m}, 1)=4 ; b_{\mathrm{m}}(\mathrm{m}, 1)=4 ; b_{\mathrm{f}}(\mathrm{m}, 1)=12 ; p(\mathrm{~m}, 1)=0 ; n(\mathrm{~m}, 2)=0 \\
& n(\mathrm{f}, 1)=10 ; n(\mathrm{f}, 2)=9 ; n(\mathrm{f}, 3)=7 ; n(\mathrm{f}, 4)=26 \\
& p(\mathrm{f}, 1)=9 ; p(\mathrm{f}, 2)=7 ; p(\mathrm{f}, 3)=6 ; p^{\prime}(\mathrm{f}, 4)=20 \\
& b_{\mathrm{f}}(\mathrm{f}, 3)=0 ; b_{\mathrm{f}}(\mathrm{f}, 4)=10 \\
& n(\mathrm{~m}, 1)=1 ; b_{\mathrm{m}}(\mathrm{m}, 2)=1 ; b_{\mathrm{f}}(\mathrm{f}, 4)=1
\end{aligned}
$$


Au contraire, en gardant le même nombre total de mâles en service, on peut abaisser ce taux d'accroissement en procédant à une réforme plus rapide des mâles. Cet effet a été démontré par HILL (1972) en considérant la taille efficace d'une population à générations chevauchantes mais sans structure en groupes; il se retrouve de façon nette pour un schéma de circulation sans décalage (Rochambeau et al., 1979, pp. 55-58; Rochambeau, 1983, pp. 147-149), mais il ne se manifeste que dans les premiers cycles de reproduction si l'on utilise un schéma avec décalage (fig. 4, cas $\mathrm{n}^{\circ}$ 2). Ces observations faites avec le modèle Poitevin sont corroborées par l'étude du modèle Mérinos Précoce, avec cependant des écarts très faibles, de l'ordre de 0,2 p. 100 sur 20 ans. Dans tous les cas le bénéfice escompté d'un renouvellement plus rapide des mâles est faible en comparaison des effets lićs au changement du système de circulation.

\section{Nombre et renouvellement des reproducteurs femelles}

L'allongement de l'intervalle de génération le long de la voie femelle-femelle peut s'obtenir en n'utilisant pour la reproduction que des femelles âgées (ayant eu au moins 3 lactations, dans le modèle Poitevin). Cette mesure se traduit par un ralentissement de la dérive génétique annuelle, de sorte que le vieillissement du cheptel femelle compense la réduction du nombre de reproductrices; l'effet en est assez faible, mais se maintient longtemps (fig. 4, cas $n^{\circ} 3$ ), et semble insensible au type de schéma de circulation (ibid.).

La réduction seule de l'effectif de femelles a un effet généralement négligeable ; dans le cas extrême du modèle Aviaire, la réduction de 16 à 4 du nombre de femelles par groupe de reproduction ne provoque qu'un faible accroissement relatif du coefficient moyen de consanguinité après 20 ans : 0,185 contre 0,150 (fig. 5).

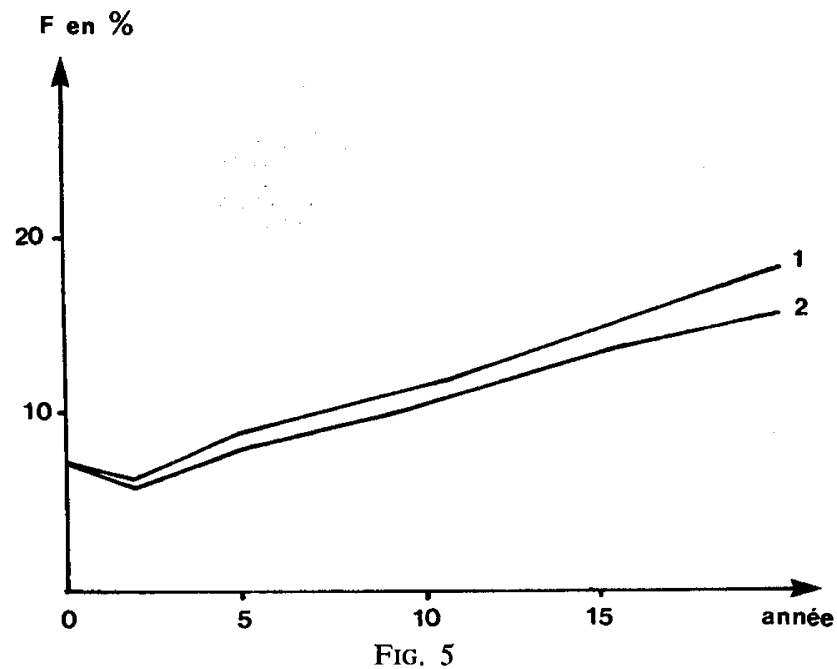

Evolution du coefficient de consanguinité moyen, dans le cas du modèle «Aviaire», en fonction du nombre de femelles par groupe de reproduction.

Change with time of mean inbreeding coefficient

in the case of the "Poultry» model population,

as function of the number of females per reproduction group.

(1) 16 femelles

16 females.
(2) 4 femelles.

4 females. 


\section{Structures génétiques initiale et induite}

Les résultats présentés ici ont été obtenus avec le modèle «Aviaire 》, pour lequel les valeurs initiales des coefficients moyens de parenté et de consanguinité présentaient une variabilité notable. Ils peuvent être transposés intégralement aux autres modèles étudiés, et semblent caractéristiques du comportement des populations gérées en groupes de reproduction. Sur le plan qualitatif, on retrouve des propriétés déjà rencontrées dans l'étude des schémas d'accouplements entre quelques individus (Wright, 1921 ; Kimura \& Crow, 1963 b ; Cockerham, 1970).

L'incidence du schéma sur la variabilité des coefficients moyens de consanguinité est illustrée par la figure 6 : le schéma avec décalage apparaît analogue aux schémas de Wright qui minimisent la consanguinité en interdisant les accouplements consanguins jusqu'à un degré fixé, qui sont connus pour provoquer une homogénéisation rapide de la consanguinité (WEIR \& CoCKerHAM, 1969).

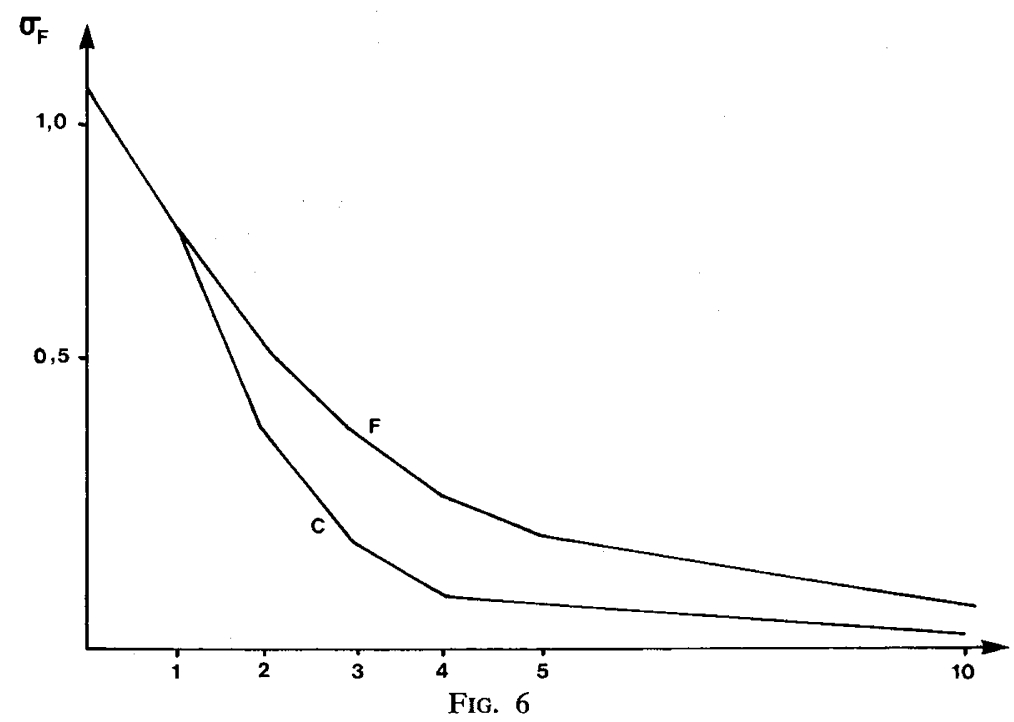

Evolution de l'écart-type du coefficient de consanguinité moyen

des femelles du modèle «Aviaire» en fonction du type de schéma de circulation des mâles avec 10 groupes de reproduction.

Change with time of the standard deviation of mean inbreeding coefficients of females, in the case of the "Poultry» model population with 10 reproduction groups.

(F) Sans décalage.

Fixed mating rule.

(C) Avec décalage.

Circular mating rule.

Les figures 7 et 8 indiquent l'importance des conditions initiales sur la structure génétique induite par un schéma. Elles représentent les valeurs des coefficients de parenté entre les mâles du groupe $\mathrm{n}^{\circ} 1$ et les animaux des autres groupes de la 

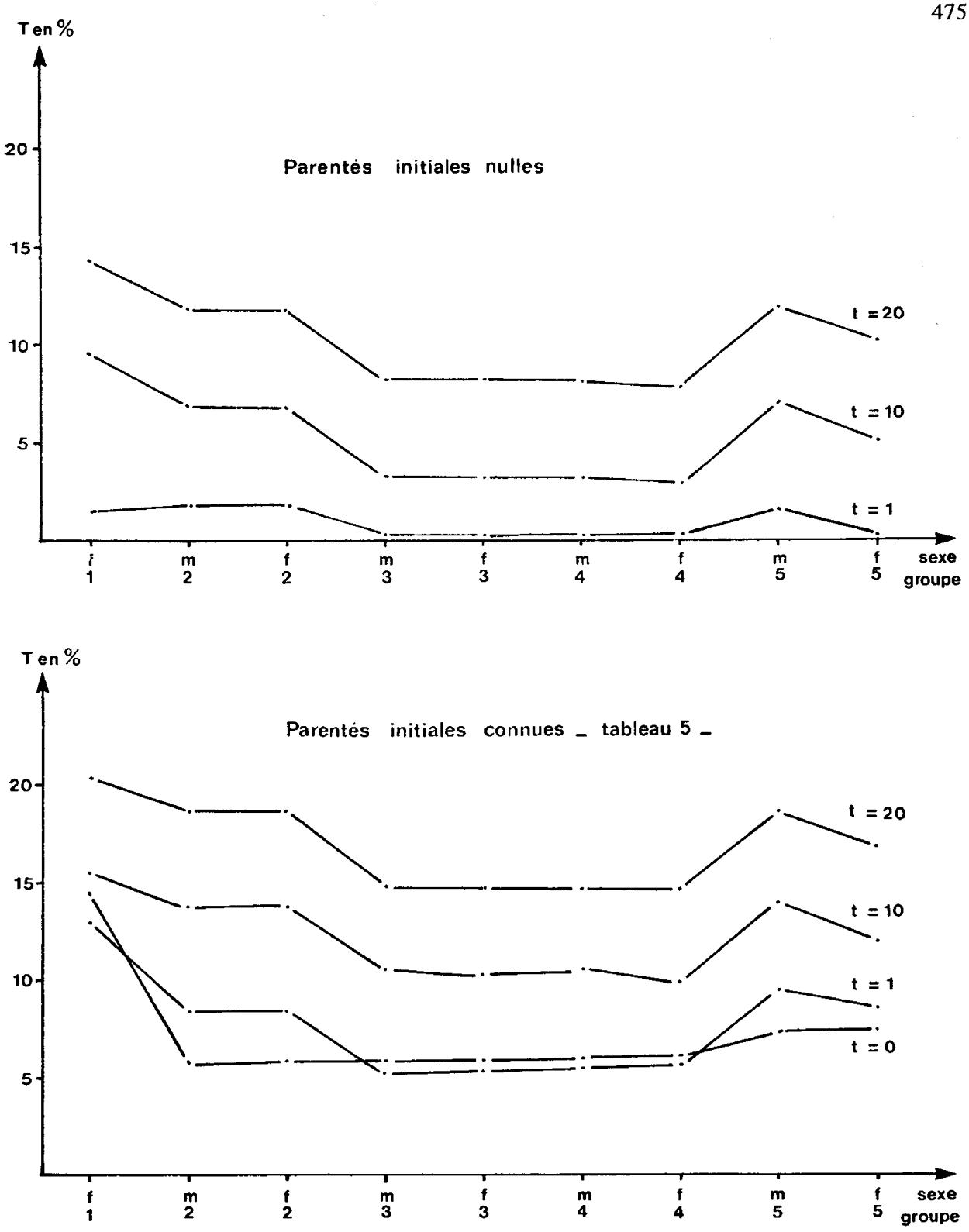

FIG. 7

Evolution du coefficient de parenté moyen, des mâles du premier groupe de reproduction avec les mâles et les femelles des autres groupes de reproduction, dans le cas du modèle "Aviaire», en fonction des coefficients de parenté initiaux pour un schéma sans décalage.

Change with time $(t)$ of mean kinship coefficient between males of the first group and other reproduction groups ( $\mathrm{m}:$ males, $\mathrm{f}:$ females), in the case of the "Poultry» model population, as dependent on initial conditions. Fixed mating rule. 

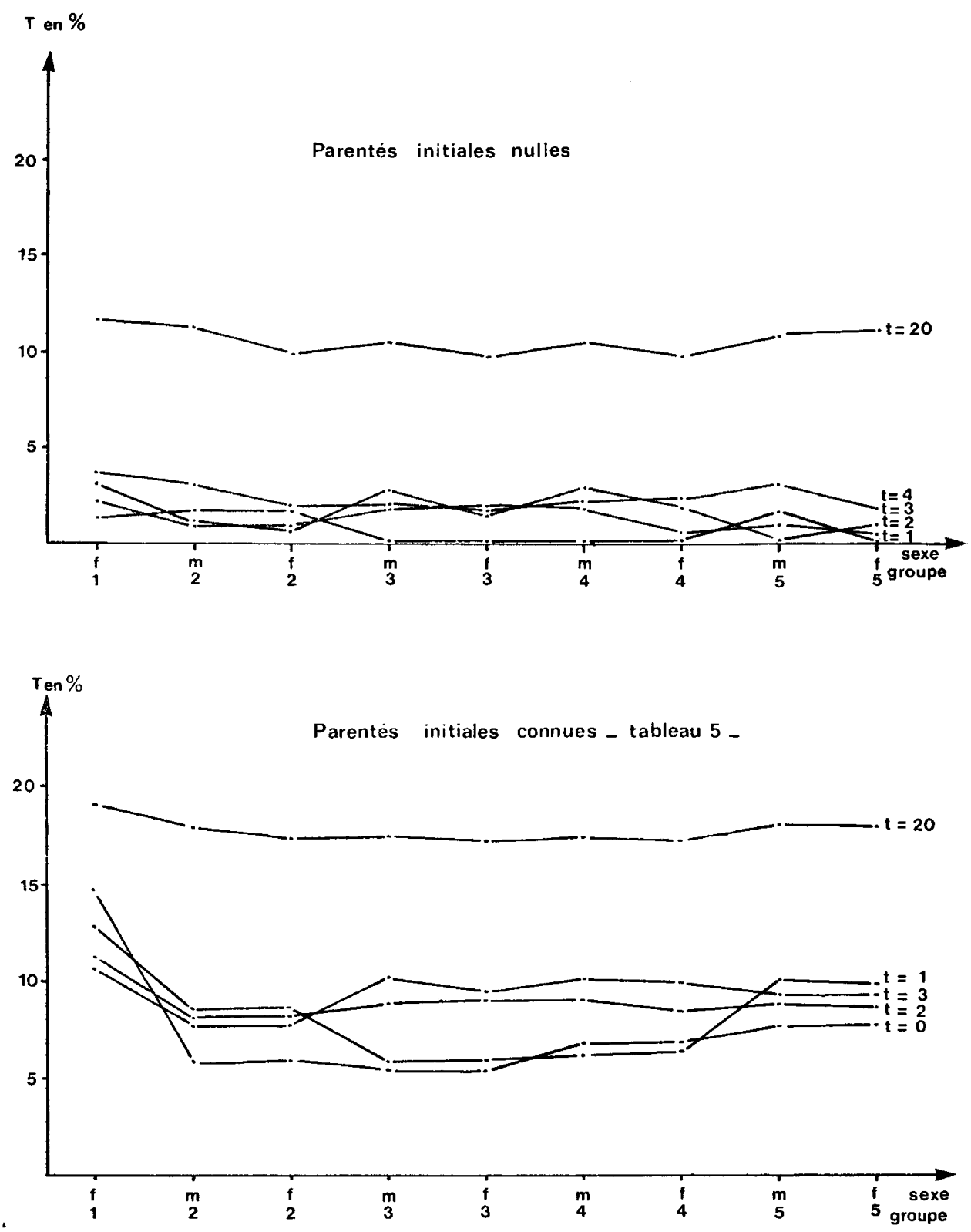

FIG. 8

Identique à la figure 7, pour un schéma avec décalage. Same as figure 7, for a circular mating rule. 
population. Les structures engendrées par les schémas sans décalage (fig. 7) sont très contrastées. Les mâles du groupe $\mathrm{n}^{\circ} 1$ présentent avec les autres groupes d'animaux des niveaux bien distincts de parenté, et ceci indépendamment des conditions initiales. Cette structure peut être prévue théoriquement pour les schémas sans décalage dans une population dont la structure démographique est constante dans le temps (mais pas nécessairement entre groupes). Dans ce cas les équations de passage d'un instant à l'autre sont caractérisées par une matrice indépendante du temps. Le vecteur propre de cette matrice associé à la plus grande valeur propre

$\left(\lambda_{\mathrm{m}}=1-\frac{1}{2 \mathrm{~N}_{\mathrm{e}}}\right.$, où $\mathrm{N}_{\mathrm{e}}$ est la taille efficace de la population) détermine des relations assymptotiques entre les coefficients de parenté : quelles que soient les conditions initiales, 2 coefficients de parenté $T(\alpha, \beta ; t)$ et $T\left(\alpha^{\prime}, \beta^{\prime} ; t\right)$ définis pour les couples $(\alpha, \beta)$ et $\left(\alpha^{\prime}, \beta^{\prime}\right)$ de groupes d'animaux à l'instant $(\mathrm{t})$ sont liés par une relation indépendante du temps, pourvu que (t) soit assez grand :

$$
\frac{1-\mathrm{T}(\alpha, \beta ; \mathrm{t})}{1-\mathrm{T}\left(\alpha^{\prime}, \beta^{\prime} ; \mathrm{t}\right)}=\underset{\alpha^{\prime} \beta^{\prime}}{\alpha \beta} \quad\left(\mathrm{t}>\mathrm{t}_{1}\right)
$$

où les constantes $\mathrm{K}$ ne dépendent que des paramètres démographiques. Après un laps de temps suffisant on peut ainsi déduire tout coefficient d'un seul, par exemple le coefficient moyen de consanguinité $F(t)$ dont l'évolution est régie par l'équation de la taille efficace :

$$
F(t)=F(t-1)+\frac{1-F(t-1)}{2 N_{e}} \quad\left(t>t_{1}\right)
$$

Les schémas avec décalage induisent en revanche une structure beaucoup plus homogène (fig. 8) qui traduit l'effet du schéma sur l'homogénéisation génétique de la population : on retrouve au niveau des coefficients de parenté entre groupes l'effet de ces systèmes d'accouplement sur l'homogénéisation des coefficients de consanguinité des individus (WeIr \& Cockerham, 1969 ; Matheron \& Chevalet, 1977). L'analyse théorique esquissée ci-dessus pour les schémas sans décalage ne peut pas être étendue, à moins de considérer l'ensemble des coefficients entre groupes définis sur un intervalle de temps égal à la période de la fonction $d$ du décalage : cette extension ne présente pas d'intérêt pratique car les temps nécessaires à l'atteinte des valeurs d'équilibre (constantes $\mathrm{K}$ ) représenteraient un très grand nombre de cycles de reproduction.

\section{Discussion}

Nous avons étudié des populations d'animaux domestiques divisées en plusieurs groupes de reproduction. Ces groupes rassemblent un ou plusieurs mâles ainsi que des femelles et ils se perpétuent par ascendance maternelle. Les reproducteurs mâles d'un groupe saillissent les femelles d'un groupe différent de celui dont ils sont issus. Dans ces conditions, 3 principes généraux se dégagent, dont le respect devrait être suffisant 
pour minimiser le taux d'accroissement de la consanguinité, compte tenu des contraintes imposées aux populations animales :

1) il faut diviser la population en au moins une dizaine de groupes de reproduction ;

2) la circulation des mâles d'un groupe de reproduction donné est programmée dans le temps de telle sorte qu'ils saillissent successivement des femelles de tous les autres groupes de reproduction;

3) le nombre de reproducteurs mâles effectivement utilisés doit être aussi élevé que possible et ils doivent être renouvelés rapidement.

Malgré la souplesse de ces quelques règles, comparée à la rigidité des schémas. théoriques conçus pour des populations de quelques individus (KImURA \& Crow, $1963 \mathrm{~b}$; YAMADA, 1980), la gestion des mâles soulève des difficultés pratiques de mise en œuvre. En monte naturelle la circulation des mâles peut être lourde : en Mérinos Précoce, l'application d'un schéma avec décalage entre 16 groupes de reproduction répartis dans 7 élevages nécessite un échange annuel d'environ 80 béliers. Malgré un léger désavantage théorique dans les premières générations, un décalage «triple » a été retenu, permettant de limiter les échanges, 2 années sur 3 , à une trentaine de béliers. L'insémination artificielle résout en principe cette difficulté, mais son usage risque d'induire un renouvellement trop lent des mâles reproducteurs, et une perte rapide des gènes de la population femelle initiale.

Les conclusions de ce travail s'appuient sur la considération d'un seul critère pour mesurer l'évolution de la variabilité génétique d'une population. D'autres approches pourraient préciser cette évolution, et contribuer au choix entre plusieurs solu. tions, équivalentes en ce qui concerne les coefficients moyens de consanguinité. Le calcul des probabilités d'origine permettrait de mesurer la divergence entre les. contenus génétiques d'une population à 2 instants : cette divergence peut s'avérer importante si les groupes de reproduction sont d'effectifs très inégaux, et le calcul des probabilités d'identité entre gènes ne permet pas de mesurer ce phénomène. D'autre part, une même probabilité d'identité moyenne entre 2 gènes peut correspondre à des structures très différentes vis-à-vis du nombre d'origines génétiques distinctes : une valeur de 10 p. 100 dans une population de 500 individus peut correspondre, en un locus, à une répartition des 1000 gènes en 10 groupes de 100 copies identiques de 10 gènes fondateurs, ou bien à 1 groupe de 300 copies d'un seuk gène, 1 groupe de 101 copies d'un autre gène fondateur, et 599 gènes copies uniques d'autant de gènes fondateurs. On conçoit que les devenirs de deux telles populations, de leurs possibilités d'être sélectionnées, ou de s'adapter à des variations d'environnement, puissent être très différents. Ce type de caractérisation des systèmes de reproduction est possible avec la description dynamique adoptée, en se restreignant. à des calculs approchés par simulation. Néanmoins l'intérêt de ces analyses complémentaires paraît plus évident dans des populations soumises à des plans de sélection intensive, pour étudier a priori l'impact des efforts de sélection et la variabilité attendue des réponses, que pour de petites populations où une gestion des accouplements avec décalage assure un brassage systématique des origines et une homogénéisation du niveau génétique.

Les résultats que nous venons de présenter concernent exclusivement la conservation d'animaux vivants, par opposition aux techniques de conservation de semence et d'embryons congelés (RENARD et al., 1983), mais la démarche utilisée est tout à 
fait générale. On peut imaginer une synthèse des diverses méthodes où l'on disposerait pour une même population d'un réservoir de gènes «congelés » et d'un échantillon d'animaux vivants permettant simultanément le renouvellement du réservoir et une adaptation minimale de la population aux variations des conditions d'élevage. Un programme de gestion du stock devrait alors être conçu, de façon à allonger artificiellement l'intervalle de génération. En considérant seulement la congélation du sperme, et en utilisant de façon différée la semence des mâles on peut établir des schémas qui échappent à la nécessité du renouvellement rapide du sexe le moins représenté (CHEvalet et al., 1984). Ces schémas demeurent moins efficaces, sur le plan théorique, que ceux que nous proposons ci-dessus en monte naturelle, mais représentent une économie importante. La prise en compte simultanée de la possibilité de congeler des embryons pourrait déboucher sur des solutions satisfaisantes également sur le plan théorique.

Reçu le 27 juin 1984.

Accepté le 28 décembre 1984.

\section{Remerciements}

Les auteurs remercient les 2 lecteurs anonymes mandatés par la revue ainsi que l'éditeur scientifique pour leurs remarques qui ont permis d'améliorer la rédaction de cet article.

\section{Références bibliographiques}

Chevalet C., De Rochambeau H., Vu Tien Khang Jacqueline, 1984. Insémination artificielle et gestion de la variabilité génétique. Séminaire des départements de génétique et physiologie animales de l'I.N.R.A., Toulouse, Auzeville, 23-24 novembre 1983, insémination artificielle et amélioration génétique : bilan et perspectives critiques. Les colloques de l'I.N.R.A., I.N.R.A., 'Versailles.

CHoY S.C., WeIR B.S., 1978. Exact inbreeding coefficients in population with overlapping generations. Genetics, 89, 591-614.

Cockerham C.C., 1967. Group inbreeding and coancestry. Genetics, 56, 89-104.

Cockerham C.C., 1970. Avoidance and rate of inbreeding. In KoJima K., Mathematical topics in population genetics, Biomathematics. Vol. 1, 104-127. Springer-Verlag, Berlin.

DEVILLARD J.M., 1980. La politique française de conservation des races domestiques en péril : premier bilan, panorama des races menacées et des programmes de conservation mis en place. B.T.I., Ministère de l'Agriculture, Paris, France. 351 et 352, 581-595.

FELSENSTEIN J., 1971. Inbreeding and variance effective numbers in populations with overlapping generations. Genetics, 68, 581-597.

Gowe R.S., Robertson A., LATTER B.D.H., 1959. Environment and poultry breeding problems. 5. The design of poultry control strains. Poult. Sci., 38, 462-471.

HILl W.G., 1972. Effective size of populations with overlapping generations. Theor. Pop. Biol., 3, 278-289.

JAmES J.W., 1972. Computation of genetic contributions from pedigrees. Theor. Appl. Genet., 42, 272-273. 
Kimura M., Crow J.F., 1963 a. The measurement of effective population numbers, Evolution, 17, 279-288.

Kimura M., Crow J.F., 1963 b. On the maximum avoidance of inbreeding. Genet. Res., 4, $399-415$.

Kimura M., Ohta T., 1971. Theoretical aspects of population genetics, $219 \mathrm{pp}$. Princeton University Press, Princeton.

Malecot G., 1972. Génétique des populations naturelles dans le cas d'un seul locus. II : Etude du coefficient de parenté. Ann. Génét. Sél. Anim., 4, 385-409.

Matheron G., Chevalet C., 1977. Conduite d'une population témoin de lapins. Evolution à court terme du coefficient de consanguinité selon le schéma d'accouplement. Ann. Génét. Sél. Anim., 9, 1-13.

Renard J.P., De Rochambeau H., Lauvergne J.J., 1983. Utilisation of gametes and embryos banking for the preservation and study of genetic resources in farm animals. Proceeding $V$ World Conference on Animal Production, 14-19 août 1983, Tokyo, Japanese Society of Zootechnical Sciences.

Robertson A., 1964. The effect of non random mating with inbred lines on the rate of inbreeding. Genet. Res., 5, 164-167.

De Rochambeau H., 1983. Gestion génétique des populations d'effectif limité : aspects méthodologiques et applications aux races d'animaux domestiques. Thèse de Docteur Ingénieur, I.N.A. Paris-Grignon.

De Rochambeau H., Chevalet C., Malafosse A., 1979. Le contrôle de la consanguinité dans les petites populations. Bull. Tech. Dép. Génét. Anim., I.N.R.A., 31.

Vu Tien Khang Jacqueline, 1983. Méthode d'analyse des données démographiques et généalogiques dans les populations d'animaux domestiques. Génét. Sél. Evol., 15, 263-298.

Weir B.S., Cockerham C.C., 1969. Group inbreeding with two linked loci. Genetics, 63, 711-742.

Wright S., 1921. Systems of mating. Genetics, 6, 111-143.

Wright S., 1922. Coefficients of inbreeding and relationship. Amer. Nat., 56, 330-338.

Wright S., 1931. Evolution in Mendelian populations. Genetics, 16, 97-159.

Wright S., 1969. Evolution and the genetics of population, Vol. 2, the theory of gene frequencies. 290 pp., University of Chicago Press, Chicago.

YAMADA Y., 1980. The importance of mating systems in the conservation of animal genetic resources. Animal genetic resources : conservation and management. F.A.O. Production and Health Papers, 24, 268-278. 